\title{
Immobilisation of a large animal for imaging purposes
}

\author{
W. Szyszkowski* and Wei Cai \\ Department of Mechanical Engineering, University of Saskatchewan, 57 Campus Drive, \\ Saskatoon, Saskatchewan, Canada S7N 5 A9
}

(Received 12 May 2008; final version received 5 March 2009)

\begin{abstract}
The mobility of a horse in the standing position with its hooves fixed to the ground is investigated to assist designing a minimally obstructing system for restraining large animals. The horse is modelled as a mechanical linkage driven by muscle forces. The linkage's configuration patterns, active joints and the forces associated with motion initiation are studied by reducing it to a mechanism with a low number of degrees of freedom. A restraint system blocking the motion of active joints with ropes is proposed as an alternative to massive mechanical devices restraining the whole body. The system has been verified experimentally on a sheep.
\end{abstract}

Keywords: standing horse; mobility; joint restraints; breaking force

\section{Introduction}

This research has been motivated by a need to build a somewhat unique restraining system to immobilise large animals for performing X-ray imaging procedures at the University of Saskatchewan synchrotron facilities (CLS). The system should provide minimally obstructed access to the whole body. The animal should be as motionless as possible for at least five minutes. No personnel (such as someone holding the halter) will be allowed to stay in the testing chamber when the high energy beamline is on. Also only mild sedation may be used, but no general anesthesia to completely paralyse the muscles. Therefore some simple devices such as metal stocks, a table and straps are not sufficient for this purpose.

We examine the possibility of completely immobilising the animal in the standing position by blocking the joints that are active during the initial phase of motion. A horse is selected for the analysis because its musculoskeletal system is relatively well known (Rooney 1981; van den Bogert 1989; Clayton 1991; Clayton et al. 2001; Hodson et al. 2001; Pilliner et al. 2002). Unfortunately, almost all research on horses has been devoted to jumping, running or walking, i.e. for the system in motion. Results from such studies do not particularly help in the design of a restraint method, which requires understanding how the horse initiates motion from rest.

In this paper a concept of mobility of standing animal (MSA) is introduced, which is related to the degrees of freedom (DOF) of the musculoskeletal system at rest. The MSA may roughly be quantified as the number of joints active when relocating certain body parts in particular patterns from the standing configuration. If the MSA is removed completely, the animal should remain motionless, a seemingly difficult task due to a relatively large number of joints (and muscles) in the whole body. However, examining the kinematics of the motion's initiation indicates that only some joints play essential roles, and that it might be sufficient to immobilise just these joints.

As already mentioned, the analyses and computer simulations were done for the horse, but preliminary tests to verify the concept were performed on sheep, which react somewhat similarly to restraints but are much easier to handle.

\section{A standing horse - assumptions and modelling}

The musculoskeletal system of a standing horse must support its weight. In particular, in a typical resting configuration all four legs carry compressive forces (approximately $60 \%$ of weight at the front and $40 \%$ at the back). The natural way of initiating motion from this configuration is to unload one leg (with the weight balanced on the remaining legs) and then raise the hoof and move it onto a new location with the motion of the whole body to follow. Therefore, the logical first step should be to fix all the hooves to the ground. Clearly this step alone does not immobilise the horse; it will only trigger its instinct to fight the restraints by using mostly the legs' mobility above the hooves and the head. Therefore the head should be immobilised next (which is relatively easy to do). The animal with the hooves/head restraints will be referred to as the partially restrained. By bending the legs, the horse with such restraints will still

*Corresponding author. Email: Walerian.Szyszkowski@usask.ca 


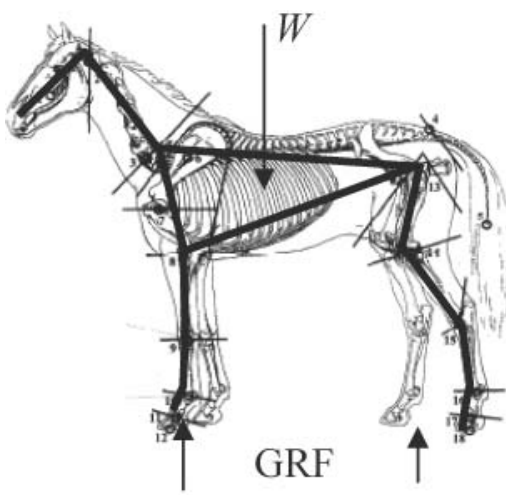

(a) The horse

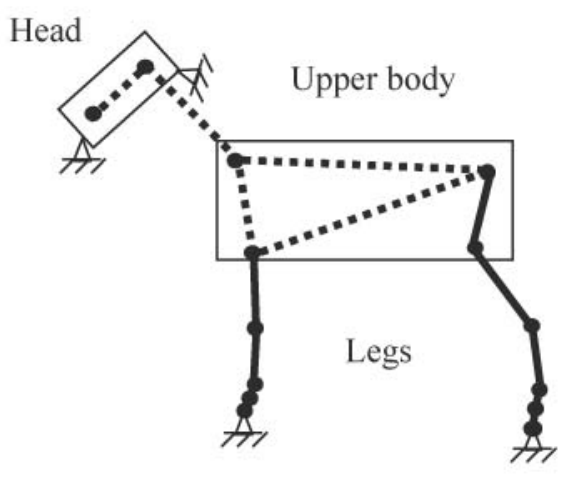

(b) The multi-link model

Figure 1. Modelling a partially restrained standing horse.

be capable of some body twisting and sideways motion in trying to free the hooves. However, if the legs are also immobilised then the horse may become immobilised sufficiently for imaging. This hypothesis is examined in the paper by estimating first the forces that might be generated to break the hoof restraints of a partially immobilised horse. Next the restraints and forces required to immobilise the legs are determined. The horse with immobilised legs will be referred to as the fully restrained.

A 26-segment horse model was adopted from Dyce et al. (1996) for the analysis and simulation (see Figure 1a). In the sagittal plane the skeletal system of a partially restrained standing horse can be represented by a 13-link planar model shown in Figure 1b. The upward ground reaction forces (GRF) compressing the legs are to balance the horse's weight $W$.

Possible movements of the legs in the sagittal plane will be examined in detail. However, the out-of-plane action needs to be considered to understand how the tensile force at the hoof can be generated (instead of compressive GRF). With the hooves fixed, the horse will try to pull one leg out of the hoof restraint. As sketched in Figure 2a, weight $W_{\mathrm{f}}$ carried by both forelimbs is distributed evenly between the two legs when the animal stands normally ( $W_{\mathrm{f}}$ will be in order of $2400 \mathrm{~N}$ for a $400-\mathrm{kg}$ horse). If the unrestrained horse tries to initiate motion with the right forelimb, for example, the weight $W_{\mathrm{f}}$ will be switched from this forelimb to the left forelimb (with the horse's weight supported by the remaining free legs) to raise the right forelimb and move forward (or backward).

With the restrained hoof the horse will use its muscles to generate tensile force $\mathrm{BF}$ (referred to as the breaking force) to pull the leg up from the ground. This force is not related to the horse's weight, but only to the strength of its muscles. The left leg will carry the compression force being approximately the sum of weight $W_{\mathrm{f}}$ and $\mathrm{BF}$, as Figure $2 \mathrm{~b}$

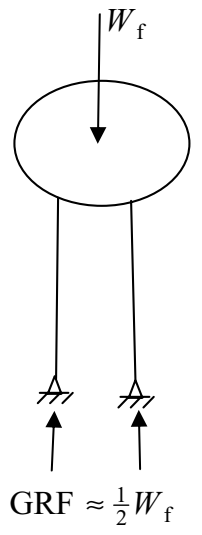

(a) Weight balanced equally

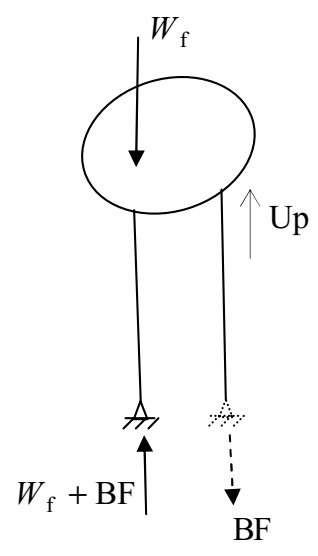

(b) Weight on one leg

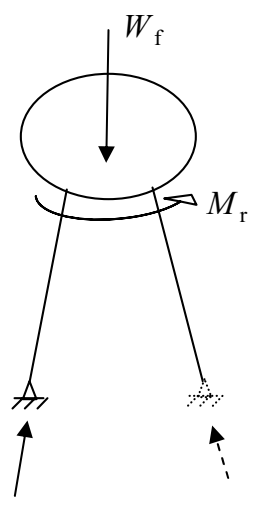

(c) Spread legs configuration

Figure 2. Weight shifting and generation of a tensile BF to free the limb. 
indicates. The leg's skeletal system is sufficiently strong to carry such a compression force without any damage, but the tensile BF can easily damage the hoof or break the hoof's restraint. Therefore, this force is the main subject of the analysis. The situation at the hindlimb is similar to the forelimb (i.e. the corresponding BF can be determined).

It should be noted that it is impossible to line up $W_{\mathrm{f}}$ vertically with the left hoof in a configuration with the legs spread (see Figure 2c). Therefore, any attempt of the horse to unload the right leg will have to be accompanied by an extra moment $M_{\mathrm{r}}$ required for the balance (otherwise the animal will fall). However, our preliminary examinations indicate that there are no sufficiently strong muscles twisting the upper body to supply such a moment, at least of the magnitude that may be needed to eliminate compression at the right leg (and further generate a tensile BF). Clearly, if all the hooves' reactions in the spread leg configuration remain compressive, the animal is not capable of fighting the restraint as effectively as in the normal straight configuration. Also, this configuration should substantially reduce swaying of the body (an effect which was observed in the experiment with the sheep). The above observations require further detailed investigations.

The rotations at joints are usually limited by the shape of articular surfaces, tension in extra capsular ligaments, contraction or passive tension in muscles and other soft structures around them. These limits, referred to as the range of motion (ROM), were taken for horses from the works by Dyce et al. (1996) and Clayton et al. (2001) and used in the analysis.

Muscle contraction is the driving force of animal locomotion. The peak isometric muscle forces are assumed in simulating the animal's fight against the restraints. The magnitudes of these forces were estimated using the method reported in Payne et al. (2005). The action of a particular muscle force is assumed to be in line with the muscle.

A computer model of the horse in Figure 1a was built using the COSMOS program and solved by the ADAMS software. Each segment was created from the 3D anatomical image. Then all 26 segments were connected at joints to generate the entire body model. This model will be referred to as anatomic (or COSMOS model) due to its geometrical similarity with the real horse. For the analysis of legs, simplified models were used in which the links were assumed as straight rigid bars with uniform averaged properties and the centre of gravity at the middle of each link. Such models (see Figure 1b) will be referred to as mechanical. Both models rendered comparable simulation results. However, the mechanical models, due to their simplicity, are easier to analyse and interpret.

\section{A partially restrained horse}

The initial MSA becomes substantially reduced by restraining the head, fixing all hooves to the ground, and by the
ROM at each joint. Nevertheless, the animal will use its muscles (and the remaining mobility) to break the hoof restraint at one leg by generating force BF (see Figure $2 b$ ), either statically (i.e. when motion and inertia effects are negligible) or dynamically (by jerking). The geometrical pattern of the limb's configuration or motion at which the maximum $\mathrm{BF}$ is generated is determined next. It is believed that the horse may be able to find such a pattern intuitively (and generate the corresponding maximum $\mathrm{BF})$.

Only motion of the limb linkage system on the sagittal plane is considered (see Figure 1b). Motion off this plane is relatively insignificant and contributes very little to fighting the restraints. Moreover, if deformability of the upper body is assumed negligible, mobility of the forelimb and hindlimb can be analysed separately.

\subsection{Forelimb's breaking force}

Major bones and joints ( Rooney 1981; Dyce et al. 1996; Pilliner et al. 2002) of a horse's forelimb are identified in Figure $3 \mathrm{a}$. These bones and joints are represented in the mechanical model by rigid links and planar revolute joints (see Figure 3b) with the rotations at each joint limited by the ROM. For comparison, the COSMOS 'anatomic' model with identical number of connected segments is presented in Figure 3c. It was built on the method described in Buchner et al. (1997). A picture of a real Dutch Warmblood horse was used to generate a model in SolidWorks (see Cai (2007) for more details).

Fixing the hoof removes mobility of the limb's bottom end, while fixing the head (see Figure 1b) restricts the upper body motion in the sagittal plane. However, the weight shifting manoeuvre sketched in Figure $2 b$ may generate up and down movements of a part of the upper body above the leg. Therefore, some vertical motion (with certain limitations to be discussed later) of the shoulder and elbow joints of the fighting leg is allowed.

Biceps, with an estimated maximal force generation capability of about 11,000 N (Payne et al. 2005), are the most powerful flexor amongst the muscles participating in fighting the forelimb's hoof restraint.

For example, lower limb extensors (such as a common digital extensor) can exert only forces that are at least 10 times smaller. Other flexors, such as deep digital flexor or superficial digital flexor, will push the fixed hoof against the ground (producing negative BF). Therefore, only the biceps action is included in the analysis to estimate the magnitude of positive (tensile) BF.

The linkage ABCD in Figure 3b, consisting of three links with 3 DOFs each (rotation and two components of translation) connected at joints $\mathrm{B}$ and $\mathrm{C}$ and constrained at D (vertically and horizontally) and at A (horizontally), has formally $3 \times 3-2 \times 2-2-1=2$ DOF. Two extra constraints are therefore needed to immobilise it. 


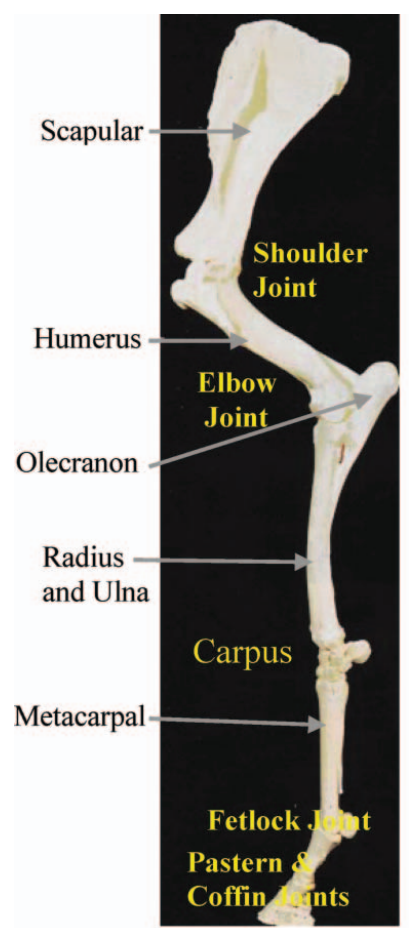

(a) Skeleton

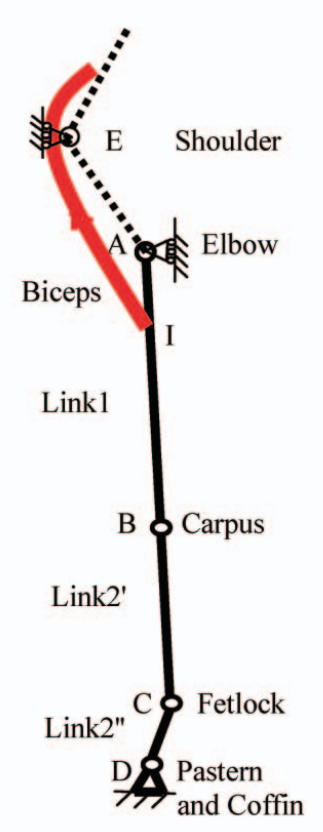

(b) Link model

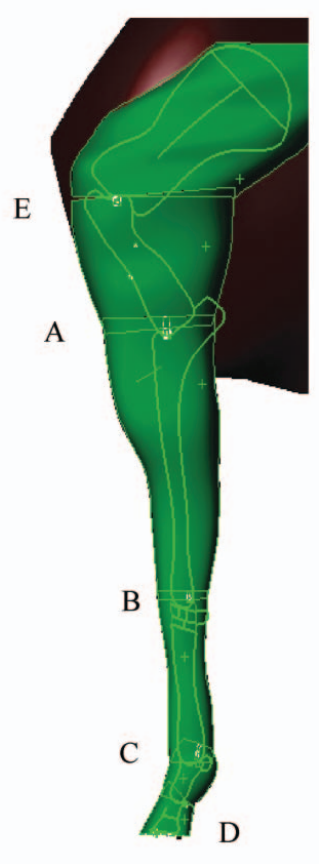

(c) COSMOS model

Figure 3. Modelling of horse forelimb.

However, Link2' and Link2" will be under tension and will align when the biceps force is applied (such a pulling configuration is indicated in Figure 4a). There will be no relative rotation at joint $\mathrm{C}$ and the system in this configuration can be simplified to a 1-DOF slider-crank mechanism driven by $F_{\mathrm{m}}$, the biceps muscle force. The link between joints $\mathrm{B}$ and $\mathrm{D}$ is of length $l_{2}=l_{2}{ }^{\prime}+l_{2}{ }^{\prime \prime}$. In the foremost stretched (vertical) configuration the elbow is at the distance $H=l_{1}+l_{2}$ from the ground (point A). Any 'pulling' configuration can be described by angles $\alpha$, or $\beta$, which in turn depend on the vertical displacement (down) of joint A, denoted as $x$. The attachment of biceps to Link1 is assumed at distance $b$ from joint A. The direction $\gamma$ of force $F_{\mathrm{m}}$ depends on distances between the biceps insertion point and the shoulder joint position denoted by $e_{x}$ and $e_{y}$ (in pulling configuration), and varies with rotation $\alpha$ of the link ( $e_{x}^{o}$ and $e_{y}^{o}$ represent the shoulder's resting position).

Taking the measurements from the COSMOS model created for an 'average' horse the following data were used in the analysis: $l_{1}=0.496 \mathrm{~m}, l_{2}=0.356 \mathrm{~m}, H=0.852 \mathrm{~m}$, $b=0.111 \mathrm{~m}, e_{x}^{o}=0.292 \mathrm{~m}, e_{y}^{o}=0.127 \mathrm{~m}$. For the data assumed, the variations of angles of $\alpha, \beta$ and $\gamma$ in degrees with the displacement $x$ are plotted in Figure 5 .

The maximum value of vertical motion $x_{\mathrm{m}}$ estimated from the COSMOS model should be smaller than about 10 $\mathrm{mm}$ at which $\beta$ reaches the ROM at the pastern and hoof joint (joint D) equal to $10.5^{\circ}$ (more explanation and details can be found in Cai (2007)). This also limits angle $\alpha$ (not to exceed $7.5^{\circ}$ ) and angle $\gamma$ (not to drop below $21.2^{\circ}$ ). First, the quasi-static case when the leg is not moving (or moving slowly so that inertia effects are negligible) under the action of force $F_{\mathrm{m}}$ is considered. Then Link 2 becomes a two-force member transmitting $\mathrm{BF}$ from $\mathrm{D}$ to joint $\mathrm{B}$, while the three forces $F_{\mathrm{m}}, \mathrm{BF}$ and $F_{\mathrm{A} y}$ exerted on Link1 must be concurrent (see Figure $4 \mathrm{c}$ ). These forces as well as the corresponding configuration can be determined by analysing equilibrium in the $x$ - and $y$-direction, and moment about $A^{\prime}$. The maximum 'static' breaking force is $\mathrm{BF}_{\mathrm{s}}=0.924 ; F_{\mathrm{m}}=10,160$ $\mathrm{N}$ at $F_{\mathrm{A} x}=0$ and $\alpha_{0}=2.5^{\circ}, \beta_{0}=3.5^{\circ}$ and $\gamma_{0}=22.7^{\circ}$ (if $F_{\mathrm{A} x}>0$ is included in the analysis then $\mathrm{BF}<\mathrm{BF}_{\mathrm{s}}$ ).

The sheep in the experiment, besides the static pulling, was observed to try fighting the hoof restraint with dynamic jerking. It is believed that the horse would behave similarly. That is, starting from the vertical (or resting) configuration the animal may try to build up momentum in all the parts that can move, by applying the biceps force $F_{\mathrm{m}}$. The maximum dynamic breaking force at the hoof, $\mathrm{BF}_{\mathrm{d}}$, will be generated when the motion is suddenly stopped at the limiting distance $x_{\mathrm{m}}=10 \mathrm{~mm}$. To analyse this case a mass $M=100 \mathrm{~kg}$ is added at the top of the limb's model to represent a portion of the upper body moving with the elbow (see Figure 6a). Also, masses $m_{1}=6.7 \mathrm{~kg}$ of Link1 


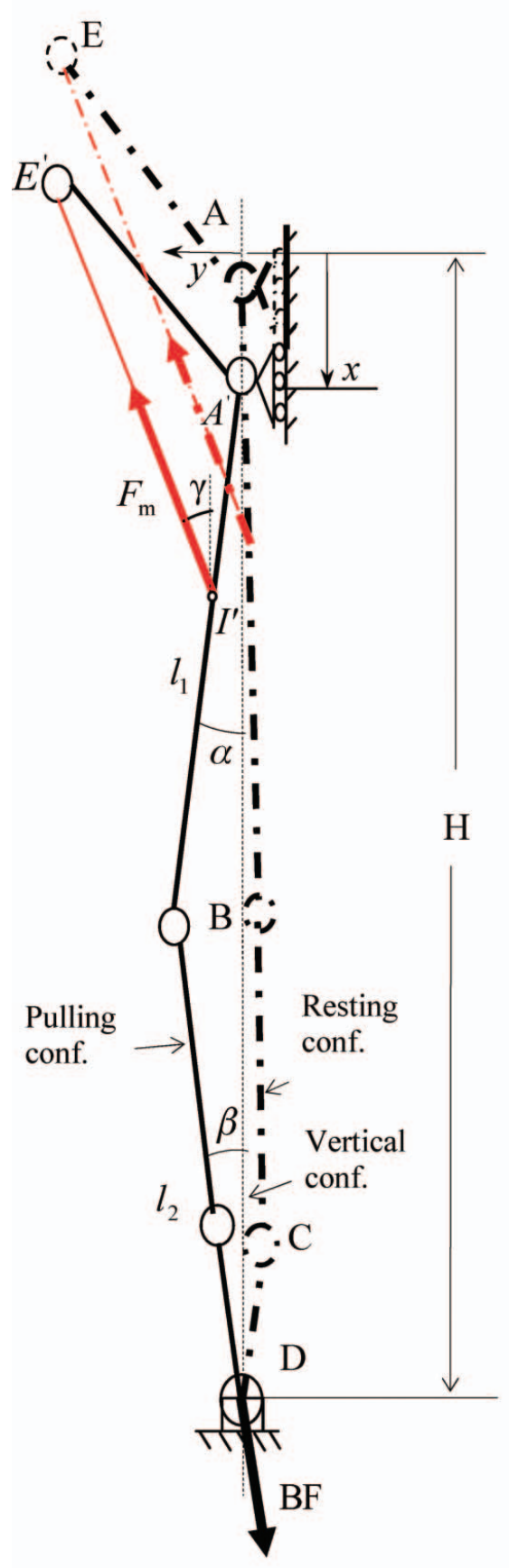

(a) Geometric configurations

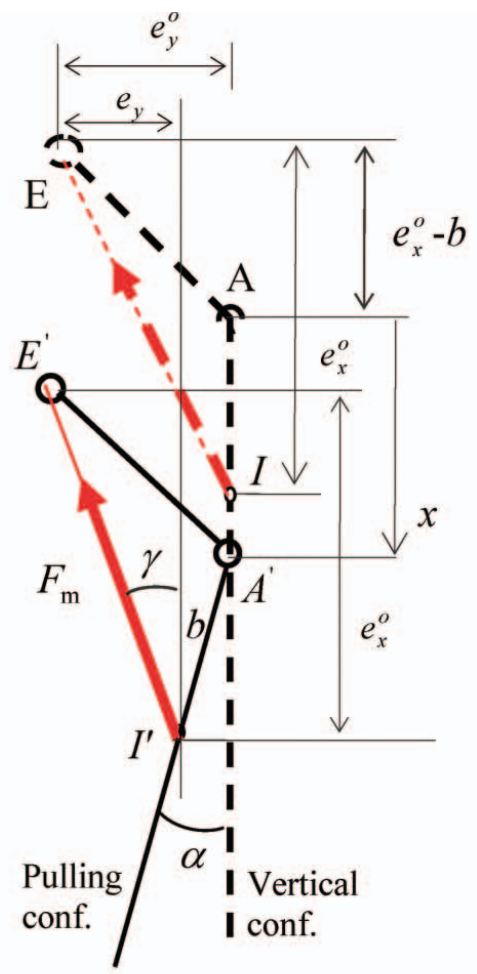

(b) Shoulder-humerus-elbow segment

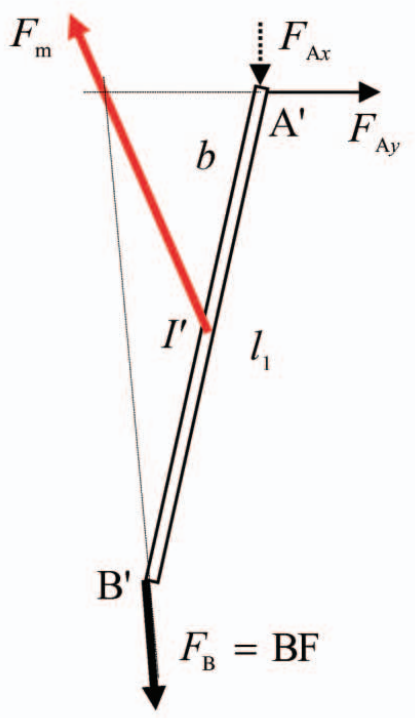

(c) Statics of Link1

Figure 4. Details of the mechanical model for static analysis.

(representing the antebrachium (Buchner et al. 1997)) and $m_{2}=2.4 \mathrm{~kg}$ of Link2 (metacarpus and proximal and middle phalanges) are included. In the mechanical model the centre of mass was placed in the middle of each link (i.e. $l_{c 1}=$ $0.5 l_{1}$ and $l_{c 2}=0.5 l_{2}$ ), while the centres in the COSMOS model were calculated automatically taking into account a specific shape of the part.

The force $\mathrm{BF}_{\mathrm{d}}$ can be estimated by formulating and solving the standard equations of motion for the model. It is assumed that the biceps force $F_{\mathrm{m}}=11,000 \mathrm{~N}$ is pulling 

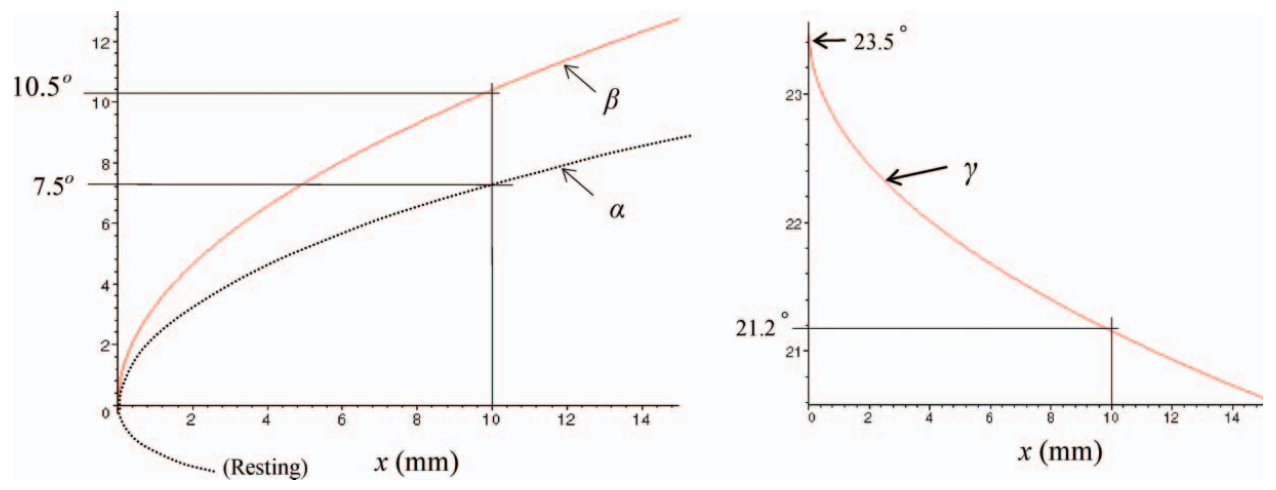

Figure 5. Range of parameters of the pulling configuration.

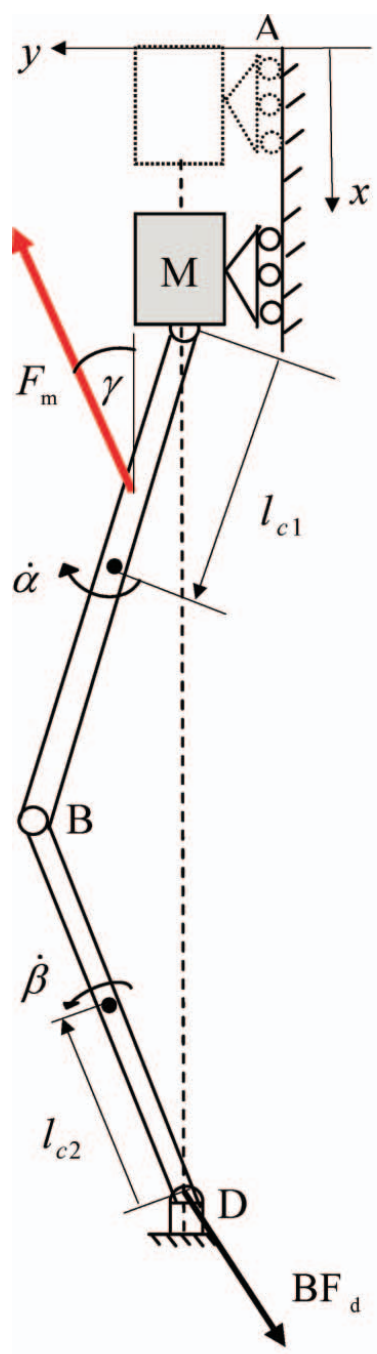

(a) The linkage
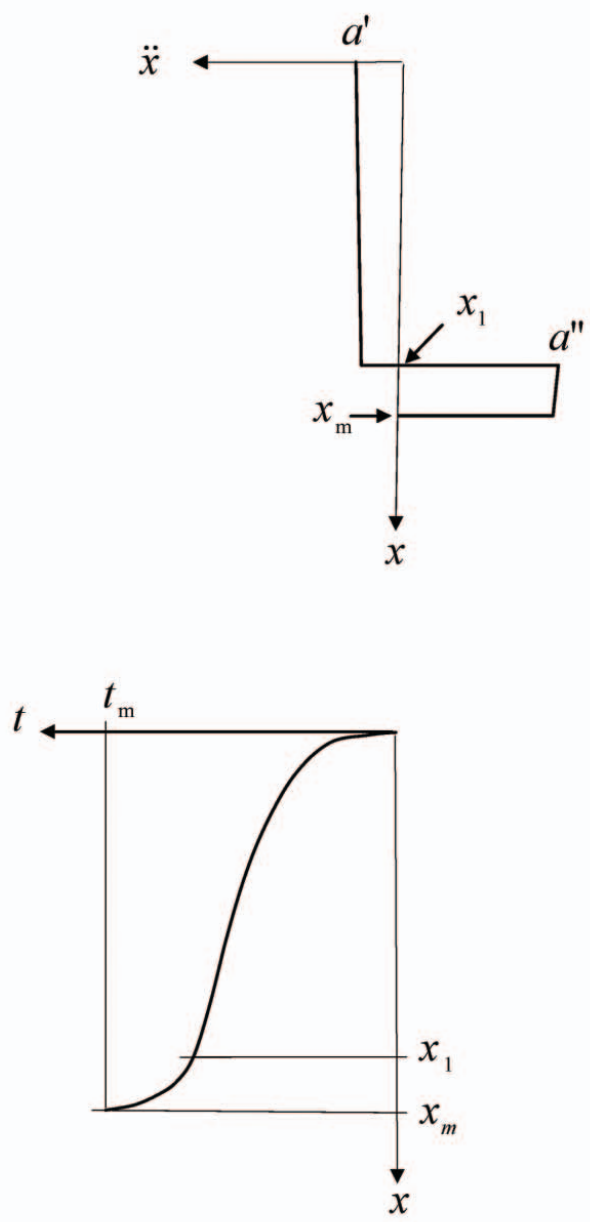

(b) Details of assumed motion of mass $M$

Figure 6. The mechanical model for dynamic analysis. 


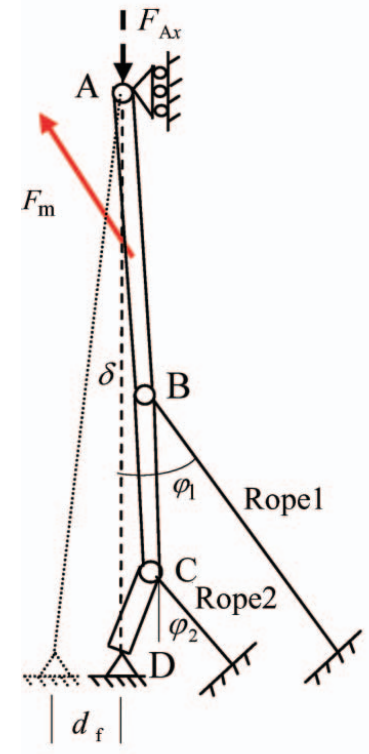

(a) Additional restraints

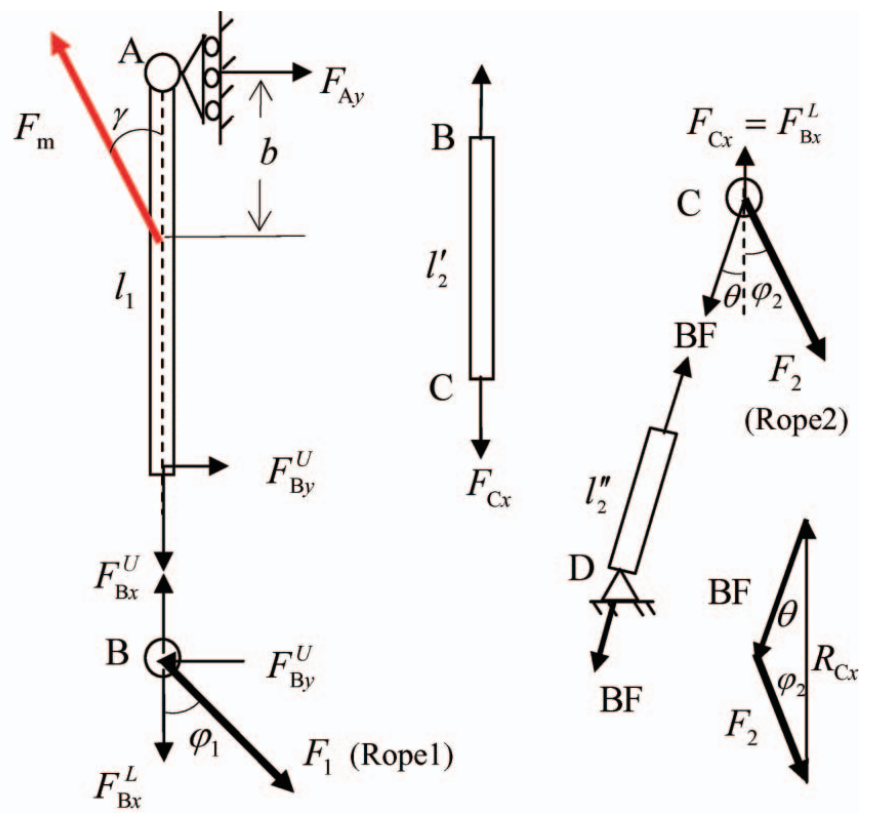

(b) Forces involved

Figure 7. The forelimb with carpal, fetlock and hoof restraints.

all the time, and that the acceleration pattern of mass $M$ is similar to the observed during the sheep restraint experiment. From the motion captured on video it has been concluded that the top of the sheep's leg was at first accelerating as free bodies fall (i.e. $a^{\prime}=\mathrm{g}$ ) and next decelerating with the rate about six times greater (i.e. $a^{\prime \prime}=-6 \mathrm{~g}$ ). The acceleration pattern and the corresponding motion pattern are shown in Figure 6b. For $x_{\mathrm{m}}=10 \mathrm{~mm}$, the acceleration switches into deceleration at $x_{1}=8.2 \mathrm{~mm}$.

The maximum $\mathrm{BF}_{\mathrm{d}}$ calculated for the mechanical model was $6718 \mathrm{~N}$, while for the COSMOS model was $6000 \mathrm{~N}$. The discrepancy was mainly attributed to different ways of calculating the inertial properties of the members in the models. Note that for the data assumed the dynamic breaking force was smaller than the static breaking force. Indeed, the sheep in the experiment was observed trying to free the forelimb from the hoof restraint mostly by a static pulling with jerking used only occasionally.

\section{Removing the forelimb mobility}

The forelimb's model of the partially restrained horse has 2 $\mathrm{DOF}$ and can generate force $\mathrm{BF}_{\max } \approx 0.92 F_{\mathrm{m}}$ (about 10,160 $\mathrm{N})$ to free the hoof. Clearly, two additional restraints are required to completely immobilise this model in the resting configuration. Two ropes, sketched in Figure 7a, to block
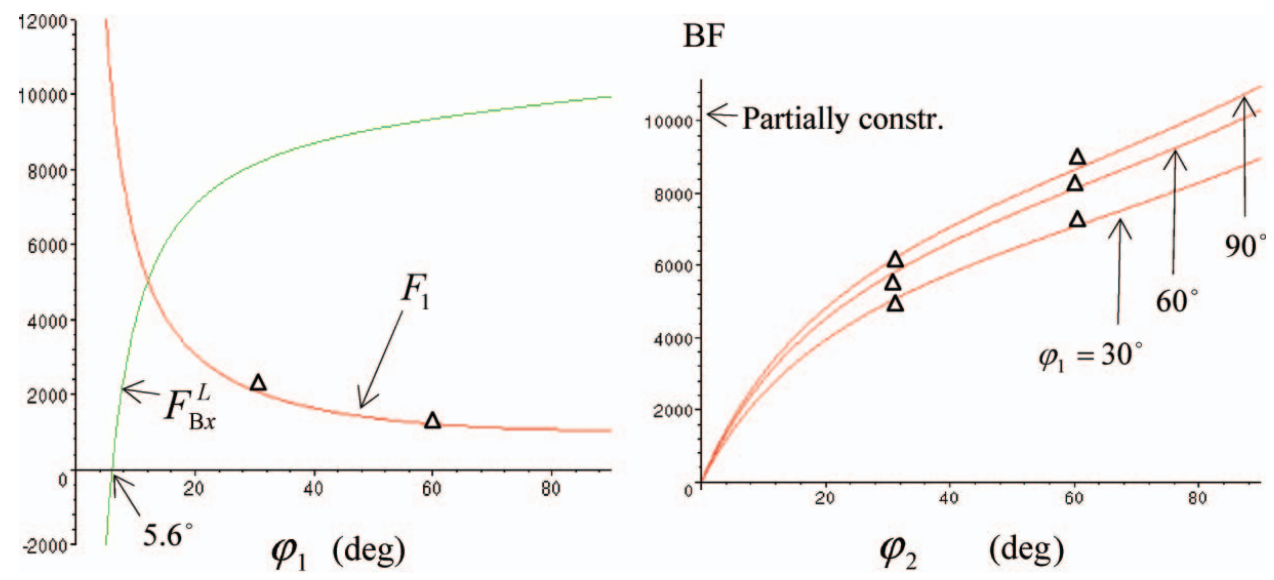

Figure 8. Forces (in newtons) in the completely restrained forelimb. 
joints $\mathrm{B}$ and $\mathrm{C}$ can be used for that purpose. The ropes should be attached to joints; otherwise the links would be subjected to undesired bending. Since motion (and jerking) is eliminated, only the static analysis is needed to obtain the forces in ropes and at the hoof. These forces are the greatest if $F_{\mathrm{Ax}}=0$ (no force is generated by the upper body).

All the forces involved (see Figure $7 \mathrm{~b}$ ) can be determined from the equations of equilibrium for particular members. Force $F_{1}$ depends on $\varphi_{1}$, while $F_{2}$ and BF depend on both $\varphi_{1}$ and $\varphi_{2}$. These forces are plotted in Figure 8 (for comparison, the same forces calculated from the COSMOS model are indicated by $\Delta$ ). If the rope angles are set more realistically to say $\varphi_{1}=\varphi_{2}=30^{\circ}$, then $F_{1}=1960 \mathrm{~N}$, $F_{2}=4430 \mathrm{~N}, \mathrm{BF}=5120 \mathrm{~N}$.

Note that BF is about $50 \%$ of the maximum BF calculated for the partially restrained leg in Section 3. This force can be further reduced if the legs are spread in the sagittal plane. For example, for $\delta=5^{\circ}$ and $F_{\mathrm{A} x} 1200 \mathrm{~N}$ the rope forces and the hoof force are $F_{1}=2050 \mathrm{~N}, F_{2}=2982 \mathrm{~N}$, $\mathrm{BF}=5000 \mathrm{~N}$.

\section{Analysis of the hindlimb}

A standing configuration of the hindlimb of a partially restrained horse is shown in Figures 9a,b. This configuration is similar to a mirror image of the forelimb's pulling configuration (see Figure 4a), and therefore the analyses of these two limbs are also similar. It is assumed that the femoris' rotation is negligible and that the biceps femoris muscle will be used to fight the hoof restraint. When data from Payne et al. (2005) are applied, the force generation capacity of this muscle is similar to the biceps muscle in the forelimb (i.e. $F_{\mathrm{m}}=11,000 \mathrm{~N}$ ). In the dynamic considerations it was assumed again that mass $M=100 \mathrm{~kg}$ is moving vertically with the stifle and hip joints. The following geometric data were adopted (see Figure 9 for the notation):

$$
\begin{aligned}
l_{1} & =0.397 \mathrm{~m}, l_{2}^{\prime}=0.302 \mathrm{~m}, l_{2}^{\prime \prime}=0.112 \mathrm{~m} \\
b & =0.211 \mathrm{~m}, e_{x}^{0}=0.305 \mathrm{~m}, e_{y}^{0}=0.239 \mathrm{~m}
\end{aligned}
$$

Similar equations of equilibrium govern the hindlimb behaviour. In the natural standing configuration $H=$ $0.783 \mathrm{~m}$ and the hoof is placed about $52 \mathrm{~mm}$ behind the vertical position of the stifle joint, which gives $\delta=3.8^{\circ}$. In this configuration the partially restrained horse (no rope) can generate statically $\mathrm{BF}_{\max }=10,340 \mathrm{~N}$ (at $\alpha=9.3^{\circ}$, $\beta=8.9^{\circ}$ and $\gamma=20.5^{\circ}$ ). If the hoof is stretched farther backward to $\delta=8.8^{\circ}$ then $\mathrm{BF}_{\max }=10,430 \mathrm{~N}$ (at $\alpha=10.2^{\circ}$, $\beta=9.8^{\circ}$ and $\left.\gamma=18.6^{\circ}\right)$. As can be seen, the hindlimb and forelimb generate similar static pulling forces.

The jerking generates $\mathrm{BF}_{\mathrm{d}}=8500 \mathrm{~N}$, which again is smaller than the static force. An important difference between the forelimb and hindlimb is that in the latter the whole limb can be immobilised by restraining the hock joint. This is because joint $\mathrm{C}$ cannot move forward due to the ROM at joint $\mathrm{D}$. Therefore only one rope at the hock as shown in Figure 9c is needed, instead of two for the forelimb. On the other hand, at this joint Link1 and Link2' are allowed to align (because of the calcaneus blocking the rotation) when the rope is tight.

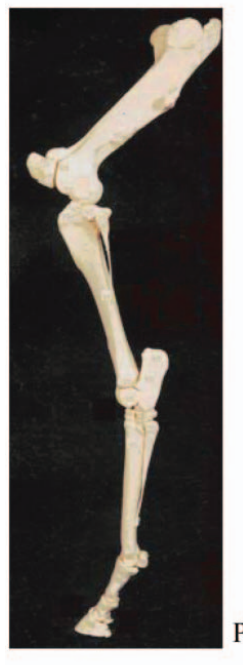

Hip joint
Stifle joint
Tarsus
Fetlock joint
stern \& coffin

(a) Skeletal structure

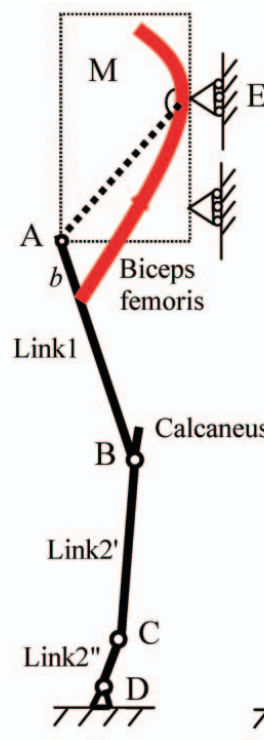

(b) Mechanical model

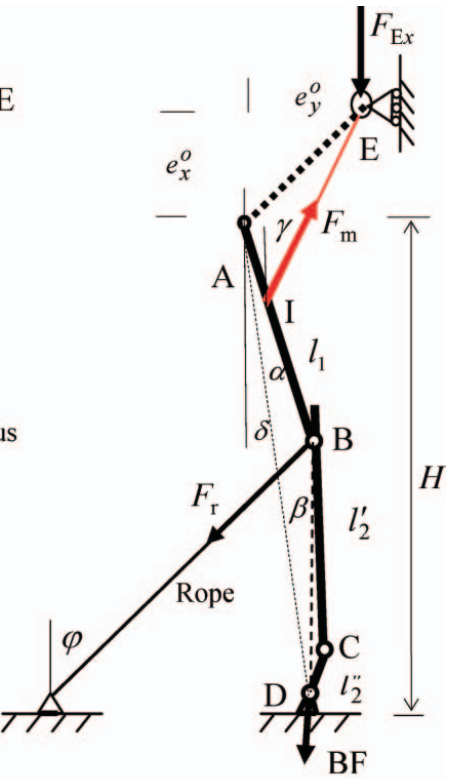

(c) Completely restrained model

Figure 9. The hindlimb. 


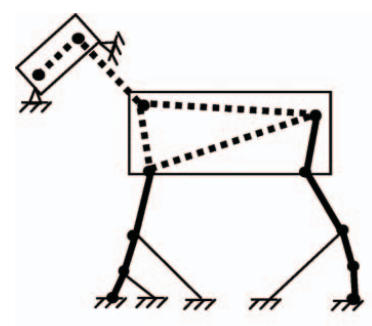

(a) Restraining a horse
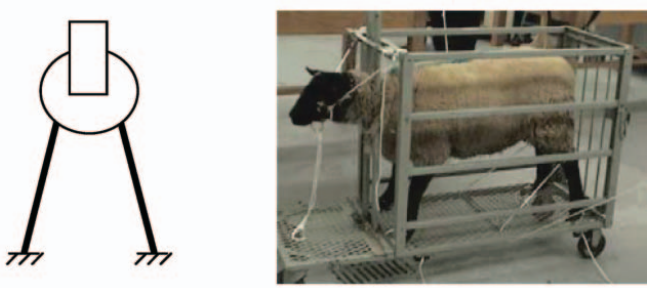

(b) Restrained sheep

Figure 10. Immobilised animals.

Such a configuration is defined by angles $\alpha=1.38^{\circ}$ and $\beta=1.32^{\circ}$. Forces $F_{\mathrm{r}}$ and BF depend on angle $\varphi$. For $\varphi=$ $30^{\circ}$ (and $\delta=8.8^{\circ}$ ) the following values were calculated: $F_{1}=5550 \mathrm{~N}$ and $\mathrm{BF}=6090 \mathrm{~N}$.

Thus the magnitude of BF drops about $42 \%$ if the leg is completely restrained. If force $F_{\mathrm{A} x}=0.2, W=800 \mathrm{~N}$ is added to represent a portion of the horse's weight carried by this leg, then the above forces modify to $F_{1}=4340 \mathrm{~N}$ and $\mathrm{BF}=6600 \mathrm{~N}$.

\section{Summary and conclusions}

The immobilisation method presented targets particular joints participating in the initiation of motion instead of blocking the body. Although each animal has a certain specific way (or ways) to move from the natural standing configuration, restraining the hooves and the head seems to be always necessary for most large animals. This permits treating the legs as low DOF linkage systems, with easily identifiable active joint, muscles, and patterns of motion. The system becomes immobilised when all the DOF are restrained, which in the case of a horse can be done with three ropes sketched in Figure 10a.

Such immobilisation provides almost unobstructed access to the whole body. Spreading the legs significantly stabilises the upper body and reduces the animal's abilities to fight the restraints. A sheep was used for a preliminary experimental verification of the method (Figure $10 \mathrm{~b})$ because of the cost and safety. As already mentioned horses and sheep have similar musculoskeletal system, and use similar strategies to initiate motion and react to restraints. The sheep's head was restrained and the hooves and joints were fixed to the bottom plate of the cart with the help of ropes and cleats (the side bars visible in the figure were never in contact with the restrained animal). The experiment, which was recorded on video, confirmed that the whole body could be immobilised if the limbs were completely restrained. The restrained sheep was standing still without any apparent discomfort for several periods lasting about five minutes each. The cart was moved around and turned without any observable movement of the body (except that related to breathing) or legs. Also, when the ropes restraining the joints were released the sheep started fighting almost immediately, and stopped when they were tightened up again.

Our computer model indicates that the above method of immobilising a sheep by restraining the joints participating in the motion's initiation should also apply to horses or other large animals. However, it must be emphasised that this conclusion is based on similarities of the mechanical aspects of such immobilisation. Additionally, these animals should also react to the required physical restraints somewhat similarly as the sheep, the assumption which is probably more realistic for bovines than for horses, and which can only be verified by carefully planned experiments on these much stronger animals.

\section{References}

Buchner HHF, Savelberg HHCM, Schamhardtt HC, Barneveld A. 1997. Inertial properties of Dutch warmblood horses. J. Biomech. 30:653-658.

Cai W. 2007. Equine immobilization with a limb restraining system [MSc thesis]. Mechanical Engineering, University of Saskatchewan.

Clayton HM. 1991. Advances in motion analysis. Vet Clin North Am: Equine Pract 7:365-382.

Clayton HM, Singleton WH, Lanovaz JL, Prades M. 2001. Sagittal plane kinematics and kinetics of the pastern joint during the stance phase of the trot. Vet Comp Orthop Traumatol. 15:1517.

Dyce KM, Sack WO, Wensing CJG. 1996. Textbook of veterinary anatomy. 2nd ed. WB Saunders. Philadelphia, WB Saunders.

Hodson E, Clayton HM, Lanovaz JL. 2001. The hindlimb in walking horses: 1. Kinematics and ground reaction forces. Equine Vet J. 33:38-43.

Payne RC, Veenman P, Wilson AM. 2005. The role of the extrinsic thoracic limb muscles in equine locomotion. J. Anatomy 206:193-204.

Pilliner S, Elmhurst S, Davies Z. 2002. The horse in motion. Blackwell Publishing, Malden, MA.

Rooney JR. 1981. The mechanics of the horse. Huntington, NY R. E. Krieger Pub. Co.

van den Bogert, AJ. 1989. Computer simulation of locomotion in the horse $[\mathrm{PhD}$ thesis]. Utrecht University. 

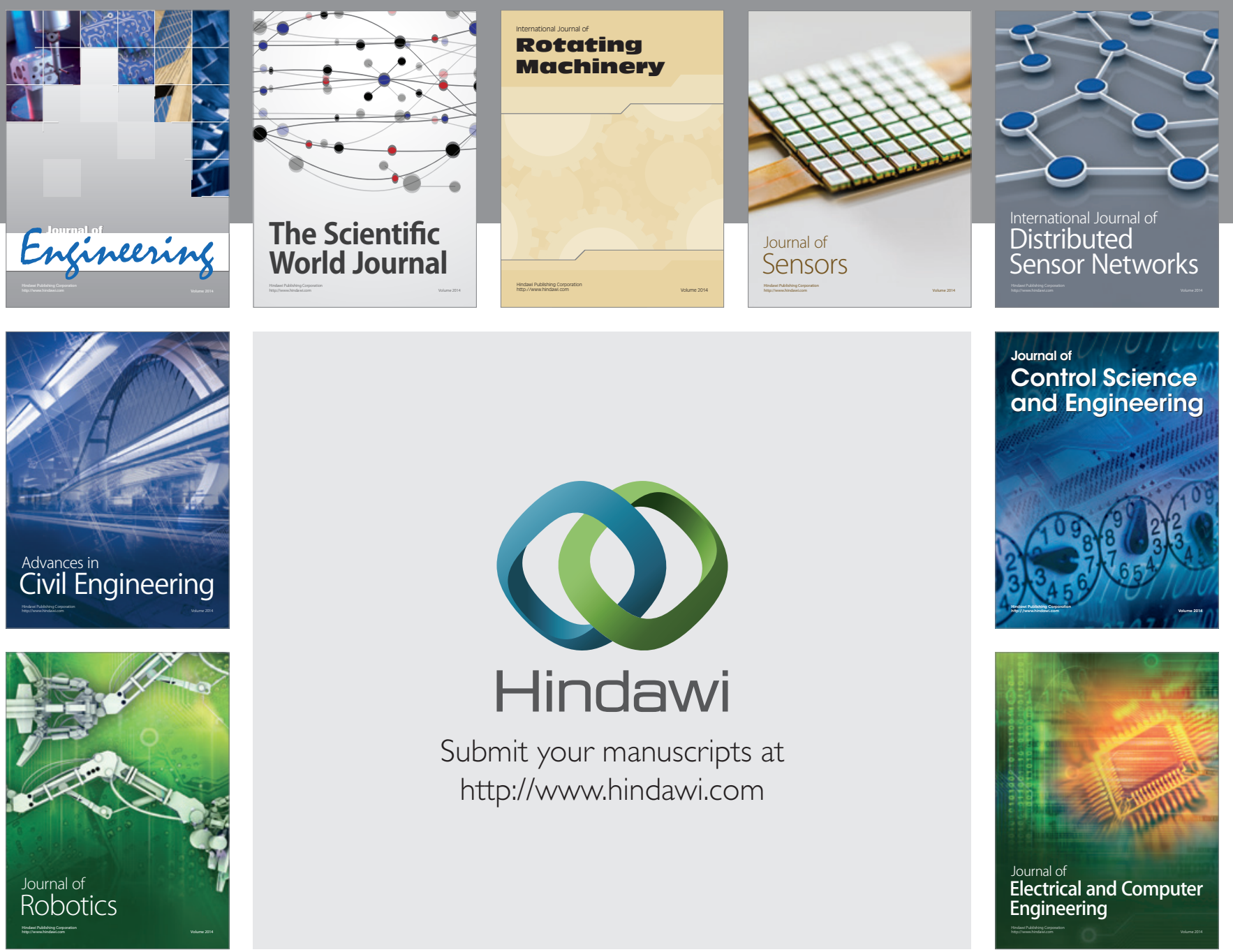

Submit your manuscripts at

http://www.hindawi.com
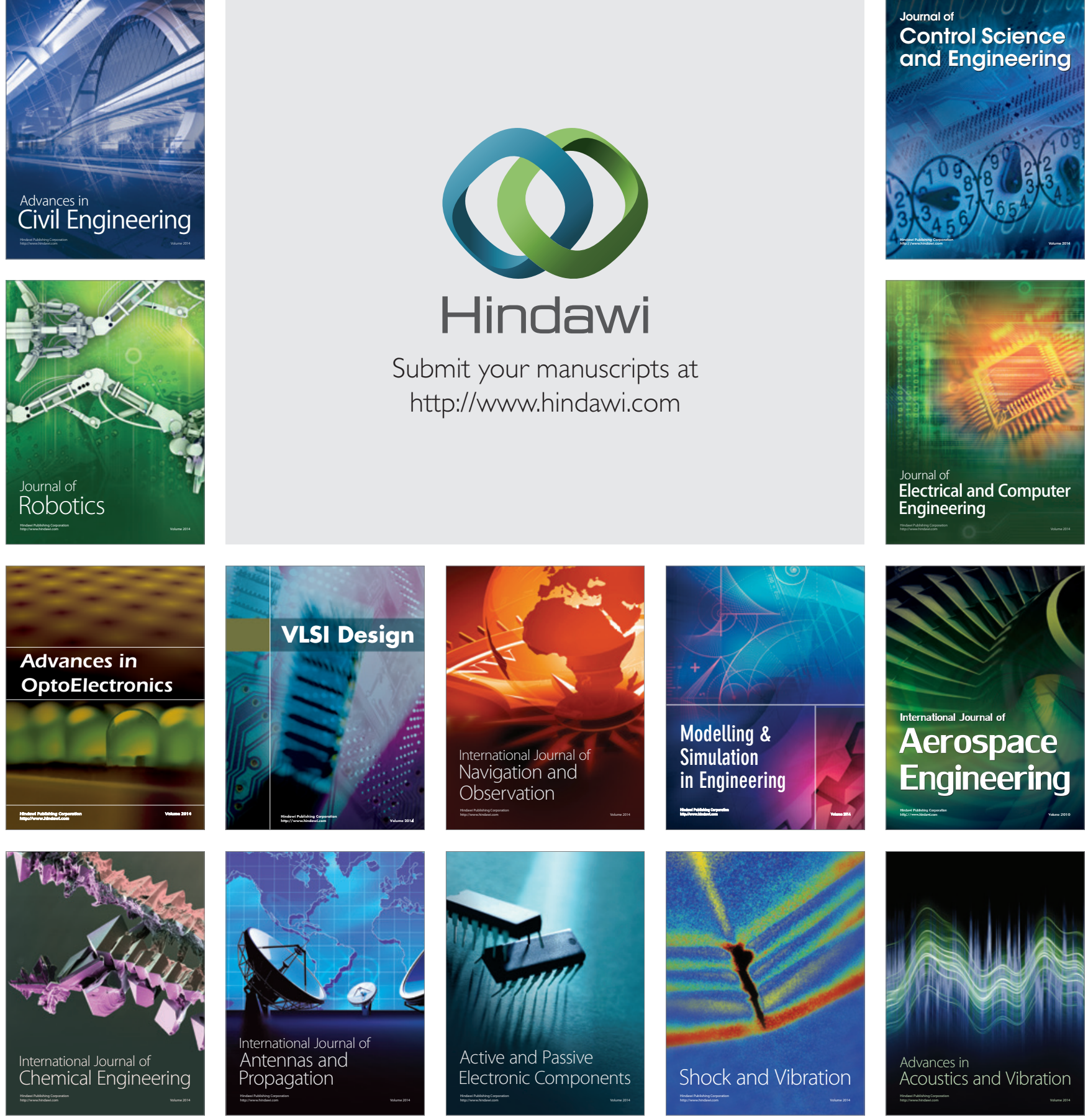